\title{
Optimization of parasite DNA enrichment approaches to generate whole genome sequencing data for Plasmodium falciparum from low-parasitemia samples
}

\section{CURRENT STATUS: ACCEPTED}

Malaria Journal $\triangle B M C$

\section{Zalak Shah}

University of Maryland School of Medicine

Matthew Adams

University of Maryland School of Medicine

Kara A Moser

University of Maryland School of Medicine

Biraj Shrestha

University of Maryland School of Medicine

Emily M Stucke

University of Maryland School of Medicine

Miriam K Laufer

University of Maryland School of Medicine

David Serre

University of Maryland School of Medicine

Joana C Silva

University of Maryland School of Medicine

Shannon Takala-Harrison

University of Maryland School of Medicine

stakala@medicine.umaryland.eduCorresponding Author

ORCiD: https://orcid.org/0000-0003-4674-8500

DOI:

$10.21203 / \mathrm{rs} .2 .17581 / \mathrm{v} 1$

\section{SUBJECT AREAS}

Infectious Diseases 


\section{KEYWORDS}

Plasmodium falciparum, malaria, whole genome sequencing, 51 selective whole genome amplification 
Abstract

Background: Owing to the large amount of host DNA in clinical samples, generation of high-quality Plasmodium falciparum whole genome sequencing (WGS) data requires enrichment for parasite DNA. Enrichment is often achieved by leukocyte depletion of infected blood prior to storage. However, leukocyte depletion is difficult in low-resource settings and limits analysis to prospectively-collected samples. As a result, approaches such as selective whole genome amplification (sWGA) are being used to enrich for parasite DNA, reducing the need for pre-processing of samples. However, sWGA has had limited success in generating reliable sequencing data from low parasitemia samples. In this study, we evaluated whether enzymatic digestion with MspJl prior to sWGA and whole genome sequencing improves genome coverage compared to sWGA alone when applied to samples representing a range of parasitemias. We also examined the potential of SWGA to cause amplification bias in polyclonal infections.

Results: MspJI digestion did not enrich for parasite DNA. Samples that underwent filtration prior to sWGA had the highest parasite DNA yield and displayed higher genome coverage compared to MspJI+sWGA and sWGA only, particularly for low parasitemia samples. The optimized sWGA approach was successfully used to generate WGS data from 218 non-leukocyte depleted field samples from Malawi. Sequences from lab-created mixtures of parasite isolates from the same geographic region generated using the optimized sWGA did not show evidence of differential amplification of parasite strains compared to directly sequenced samples.

Conclusion: The optimized sWGA approach is a reliable method to obtain WGS data from nonleukocyte depleted, low parasitemia samples. The absence of amplification bias in data generated from mixtures of isolates from the same geographic region suggests that this approach can be appropriately used for molecular epidemiological studies.

\section{Full-text}

Due to technical limitations, full-text HTML conversion of this manuscript could not be completed. However, the manuscript can be downloaded and accessed as a PDF.

Figures 

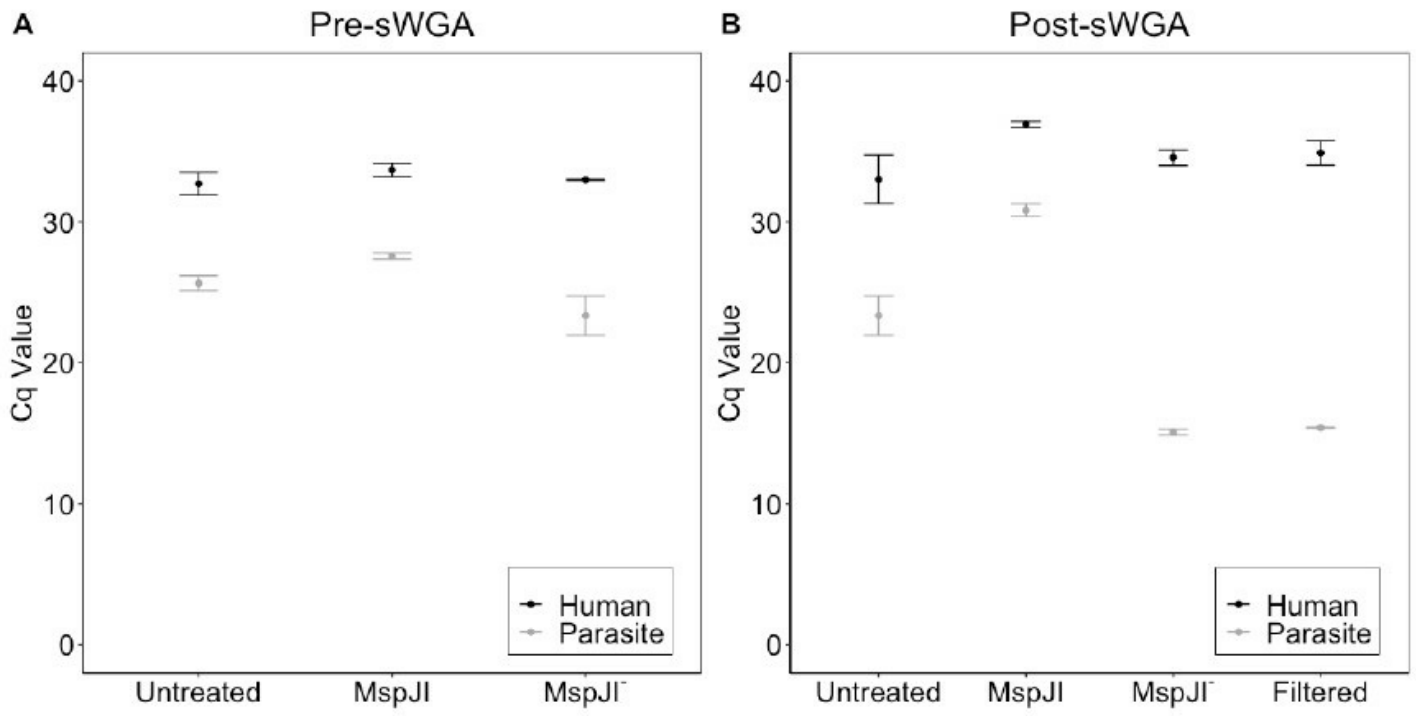

Figure 1

Figure 1

Effect of MspJI and sWGA treatments on parasite DNA yield. (A) Human and P. 418 falciparum Cq values prior to sWGA on samples with 10,000 parasites/ $\square \mathrm{L}(\mathrm{n}=3)$. Cq value 419 indicates the number of cycles required to detect a signal, where higher Cq values indicate 420 lower DNA concentrations. (B) Human and P. falciparum Cq values after sWGA on samples with 421 10,000 parasites/ $\square \mathrm{L}(\mathrm{n}=3)$. 

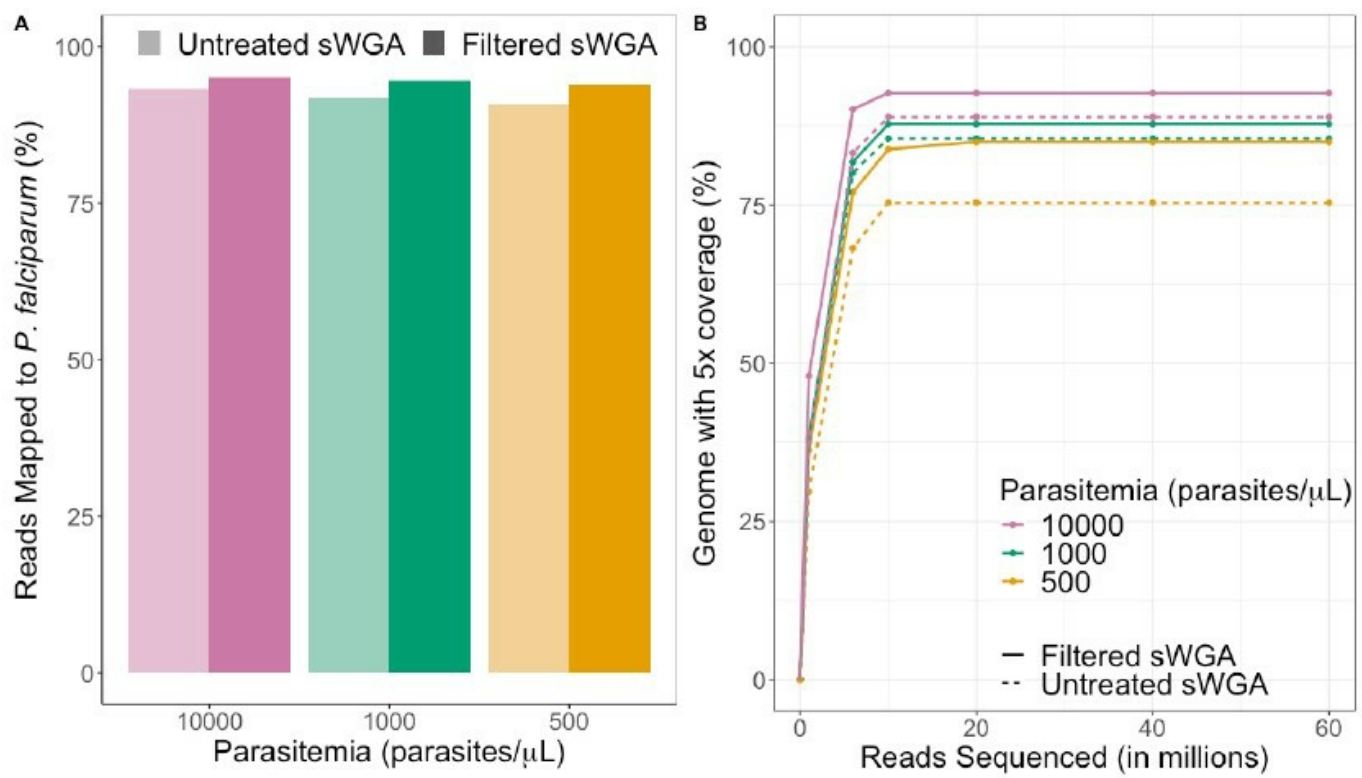

Figure 2

Figure 2

P. falciparum genome coverage in filtered and untreated samples that underwent 424 sWGA. (A) The percentage of reads that mapped to the P. falciparum 3D7 reference are shown 425 for filtered and untreated samples with different parasitemias that underwent sWGA prior to 426 sequencing. (B) Percentage of the P. falciparum 3D7 genome with at least $5 x$ coverage is shown 427 relative to the number of reads sequenced (in millions) in filtered and untreated samples of 428 different parasitemias that underwent sWGA prior to sequencing. 


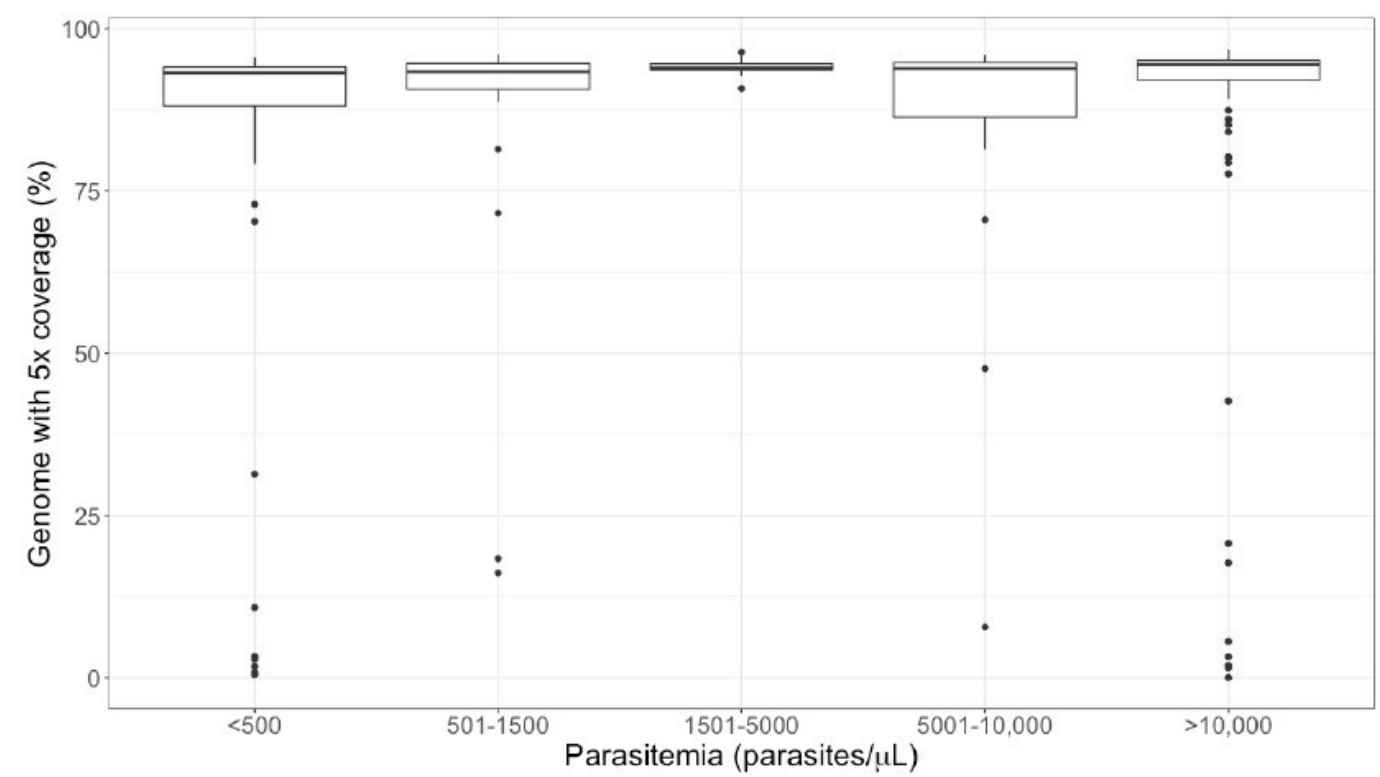

Figure 3

Figure 3

Percent of genome with $5 x$ coverage in whole genome sequences of field isolates 431 with different parasitemias.

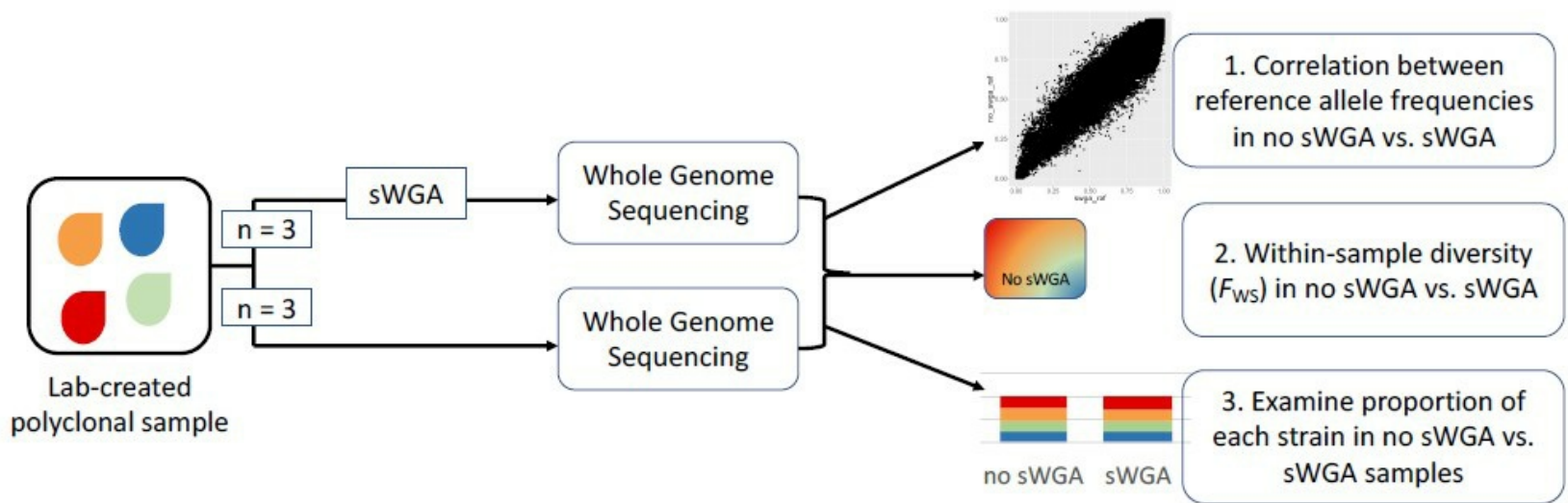

Figure 4

Figure 4

Schematic of experimental design to evaluate potential amplification bias in samples 434 undergoing sWGA. 


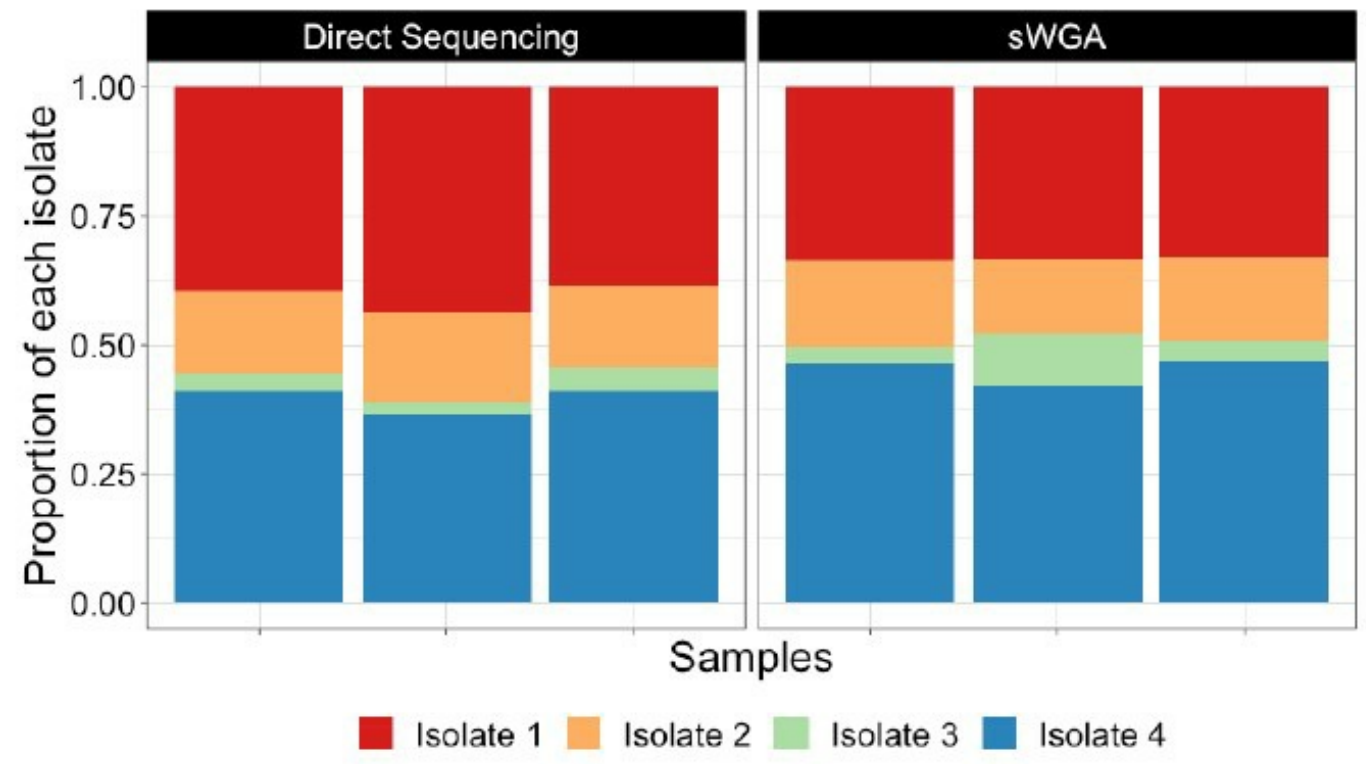

Figure 5

Figure 5

Proportion of isolate-specific SNPs in mixtures that underwent sWGA prior to 437 sequencing or were directly sequenced.

\section{Supplementary Files}

This is a list of supplementary files associated with this preprint. Click to download.

AdditionalFile1.pdf 